OPEN ACCESS

Edited by:

Danuta Radzioch,

McGill University, Canada

Reviewed by:

Binqing $\mathrm{Fu}$,

University of Science and Technology

of China, China

Haiming Wei,

University of Science and Technology

of China, China

*Correspondence:

Xiaofeng Xu

xxf0550@126.com

Yunxia Cao

caoyunxia6@126.com

Xiangyan Wang

wangxiangyan0429@163.com

Huijuan Zou

hienjoyshine@aliyun.com

${ }^{+}$These authors have contributed equally to this work

Specialty section:

This article was submitted to Cytokines and Soluble Mediators in Immunity, a section of the journal

Frontiers in Immunology

Received: 24 September 2021 Accepted: 06 December 2021

Published: 23 December 2021

Citation:

Zhu D, Zou H, Liu J, Wang J, Ma C,

Yin J, Peng $X$, Li D, Yang Y, Ren Y,

Zhang $Z$, Zhou $P$, Wang $X$, Cao $Y$ and

$X u X$ (2021) Inhibition of HMGB1

Ameliorates the Maternal-Fetal

Interface Destruction in Unexplained

Recurrent Spontaneous Abortion by

Suppressing Pyroptosis Activation.

Front. Immunol. 12:782792.

doi: 10.3389/fimmu.2021.782792

\section{Inhibition of HMGB1 Ameliorates the Maternal-Fetal Interface Destruction in Unexplained Recurrent Spontaneous Abortion by Suppressing Pyroptosis Activation}

\author{
Damin Zhu ${ }^{1,2,3,4 \dagger}$, Huijuan Zou ${ }^{1,3,4 * t}$, Jinxian Liu ${ }^{1,5,6,7}$, Jing Wang ${ }^{1,5,6,7}$, Cong Ma ${ }^{1,5,6,7}$, \\ Jiaqian Yin ${ }^{1,5,6,7}$, Xiaoqing Peng 1,5,6,7, Danyang Li $^{1,5,6,7}$, Yulu Yang ${ }^{1,5,6,7}$, Yu Ren ${ }^{1,5,6,7}$, \\ Zhiguo Zhang ${ }^{1,5,6,7}$, Ping Zhou ${ }^{1,5,6,7}$, Xiangyan Wang ${ }^{2 *}$, Yunxia Cao ${ }^{1,3,4 *}$ \\ and Xiaofeng $X u^{1,3,4 *}$

\begin{abstract}
${ }^{1}$ Reproductive Medicine Center, Department of Obstetrics and Gynecology, the First Affiliated Hospital of Anhui Medical University, Hefei, China, ${ }^{2}$ Department of Obstetrics and Gynecology, Chaohu Hospital of Anhui Medical University, Chaohu, China, ${ }^{3}$ National Health Commission Key Laboratory of Study on Abnormal Gametes and Reproductive Tract (Anhui Medical University), Hefei, China, ${ }^{4}$ Key Laboratory of Population Health Across Life Cycle (Anhui Medical University), Ministry of Education of the People's Republic of China, Hefei, China, ${ }^{5}$ Anhui Province Key Laboratory of Reproductive Engineering Research Center, Anhui Medical University, Hefei, China, ${ }^{7}$ Anhui Provincial Institute of Translational Medicine (Anhui Medical University), Hefei, China
\end{abstract} \\ Health and Genetics (Anhui Medical University), Hefei, China, ${ }^{6}$ Biopreservation and Artificial Organs, Anhui Provincial
}

Recurrent spontaneous abortion (RSA) is a common complication of pregnancy that affects the physical and mental health of pregnant women, and approximately $50 \%$ of the mechanisms are unclear. Our previous studies have found that high mobility group box 1 (HMGB1) molecules are highly expressed the maternal-fetal interface of unexplained recurrent spontaneous abortion (URSA) patients. The purpose of this study was to further detect the expression of HMGB1 and pyroptosis in decidual tissue of URSA patients, and explore the potential mechanism of the protective role of HMGB1 in URSA patients and mouse model. The decidua tissues of 75 URSA patients and 75 women who actively terminated pregnancy were collected, and URSA mouse models were established and treated with HMGB1 inhibitor-aspirin. The expression of HMGB1, and their receptors (RAGE, TLR2, TLR4), pyroptosis-associated proteins (NLRP-3, caspase-1, GSDMD) and $\mathrm{NF}-\kappa \mathrm{B}$ was examined at the maternal-fetal interface of human and mouse. Our study found that HMGB1, NLRP-3, Caspase-1, GSDMD, RAGE, TLR2 and TLR4 were highly expressed and NF-kB signaling pathway were activated in the decidua tissue of URSA group. Moreover, immune cell disorder and co-localization of HMGB1 and macrophages were found at the maternal-fetal interface of URSA mice. However, HMGB1, TLR2, TLR4, $\mathrm{NF}-\mathrm{\kappa B}$, and pyroptosis-associated proteins can be down-regulated by administering lowdose aspirin. These data may indicate that highly expressed HMGB1 was actively 


\begin{abstract}
secreted by macrophages and then activated pyroptosis through the TLR2/TLR4-NF- $\mathrm{BB}$ pathway to cause aseptic inflammation, leading to the occurrence and development of URSA. Moreover, low-dose aspirin can reduce HMGB1 protein levels of serum and decidual in URSA.
\end{abstract}

Keywords: maternal-fetal Interface, high mobility group B-1, macrophage, pyroptosis, URSA

\section{INTRODUCTION}

Two or more consecutive or discontinuous losses of pregnancy products before 24 weeks with the same sex partner are referred to as recurrent spontaneous abortion (RSA) or recurrent pregnancy loss (RPL) $(1,2)$. In recent years, the incidence of RSA has been relatively high, affecting approximately $1 \%$ of women of childbearing age (3). The risk of RSA also increases further with the number of miscarriages, reaching about $40 \%$ after three consecutive miscarriages, and the prognosis is poor with increasing age (4). This not only affects the physical and mental health of pregnant women, but also brings heavy burdens and mental pressure to their families. The etiology of RSA include genetic abnormalities, structural abnormalities, infection, endocrine abnormalities, immune dysfunction, antiphospholipid syndrome, and thrombophilic disorders, etc (5). However, the causes in nearly $50 \%$ of RSA patients are still unclear, and the clinical diagnosis is called unexplained recurrent spontaneous abortion(URSA) (6). Fetuses as allografts are able to grow and develop normally without rejection within the mother, which depends on the process of a constant reciprocal balance of maternal immune rejection or immune tolerance to the fetus (7). Abnormal immune tolerance at the maternal-fetal interface could be one of the important pathogenesis of URSA (8).

High mobility group box-1 (HMGB1) is a highly conserved non-histone DNA-binding protein widely distributed in the nucleus of eukaryotic cells, characterized by the low molecular weight and rapid mobility in the gel electrophoresis $(9,10)$. HMGB1, a typical damage-associated molecular pattern (DAMP) molecule, can be actively or passively released into the extracellular space (10), which binds to its receptor ligands RAGE, TLR, CXCR4, TIM-3, CD24, NMDAR, and haptoglobin to exert the proinflammatory role $(11,12)$. Inflammation plays an important role in embryo implantation, development, and endometrial decidualization (13). Low level of HMGB1 can cause the physiological inflammatory response, recruit immune cells, and promote the proliferation and differentiation of decidual stromal cells that are required for nomal pregnency (13). However, high levels of HMGB1 in the uterine cavity were associated with pregnancy failure (13). Recent studies have found that HMGB1 was highly expressed in villous and decidual tissues of RSA patients $(14,15)$, moreover, animal experiments have also shown that excessive administration of HMGB1 leaded to the failure of pregnancy (16).

Pyroptosis, which has been extensively studied recently, is a lytic and inflammatory programmed cell death pathway different from apoptosis (17). It is involved in the occurrence and development of a variety of diseases in multiple organs of the body. Pyroptosis directly assembles the inflammasome (NLRP3) by damage-associated molecular pattern (DAMP) and activates the cysteine protease caspase-1, allowing the translocation of the $\mathrm{N}$-terminal domain fragment of the GasderminD (GSDMD) protein to the cell membrane to form $\beta$-barrel transmembrane pores (17). Hence, the plasma membranes were rapidly ruptured to release cellular contents and a large number of inflammatory factors, and send proinflammatory signals to adjacent cells to recruit inflammatory cells and induce an inflammatory response, eventually causing cell death (18).

HMGB1 induced hepatocyte pyroptosis has been shown to lead to aseptic inflammation and liver injury (19). But the pyroptosis mediated by HMGB1 has never been investigated in URSA patients. Moreover, our previous studies have found that the decidua tissue of URSA patients highly expresses HMGB1 (20), and the peripheral blood HMGB1 expression of URSA patients taken aspirin of the most effective inhibitors of HMGB1 is decreased $(15,21)$. However, the specific mechanism is not clear. Therefore, this study aimed to investigate the release mechanism of HMGB1 in URSA and explore the effect of blocking HMGB1 on URSA. This study would provide a theoretical basis for clinical treatment of URSA patients.

\section{MATERIALS AND METHODS}

\section{Human Sample}

From September 2017 to December 2020, 140 pregnant women who were younger than 35 years old with a history of two or more unexplained miscarriage were recruited from the Department of Obstetrics and Gynecology of the First Affiliated Hospital of Anhui Medical University in this study. Exclusion criteria included anatomical abnormalities of the genital tract, endocrine abnormalities, immune abnormalities, coagulation abnormalities, karyotype abnormalities in couples, autoantibody abnormalities such as anticoagulant lipid antibodies, thyroid dysfunction, genetics, and infection. Pregnant women underwent a vaginal ultrasound examination every two weeks. When the fetal heart rate was found to disappear, it was defined to terminate the pregnancy and a villus chromosome test was performed. Decidua tissues during artificial abortion were then collected, and only 75 patients were enrolled for follow-up study after excluding the decidua tissues with abnormal chorionic chromosome detection. In the control group, 75 pregnant women from the same hospital voluntarily were chosen to terminate the pregnancy by abortion, from which the decidual tissue were collected. The biological sample study was approved by the Medical Ethics Committee of the First 
Affiliated Hospital of Anhui Medical University (Ethics approval number: PJ2018-02-09; Clinical Trial Registration number: ChiCTR1800015403).

\section{Animals}

Female CBA/J mice and male DBA/2J mice (8-10 weeks old) were purchased from Beijing Huafukang Biotechnology. Male $\mathrm{BALB} / \mathrm{c}$ mice (8-10 weeks old) were purchased from the Experimental Animal Center of Anhui Medical University. All mice were adaptively fed for one week at a standard environment of constant temperature of $21-23^{\circ} \mathrm{C}$ and humidity of $50-60 \%$, with autoclaved sterile water and diet. All animal experiments were controlled specific pathogen-free conditions at the Experimental Animal Center of Anhui Medical University. All experimental protocols were approved by the Experimental Animal Ethics Committee of Anhui Medical University (ethics number: LLSC20201138).

\section{Establishment and Intervention of URSA Mouse Model}

All female mice were randomly divided into three groups. Female $\mathrm{CBA} / \mathrm{J}$ mice mated with male $\mathrm{BALB} / \mathrm{c}$ mice as the control group. Female $\mathrm{CBA} / \mathrm{J}$ mice mated with male DBA/2J mice as the URSA group. And aspirin-treated female CBA/J mice mated with male DBA/2J mice as the aspirin treatment group (URSA+ASPL). Female mice in the aspirin treatment group were given aspirin-containing drinking water $(30 \mathrm{ug} / \mathrm{ml})$ for 7 days before mating and 7 days after vaginal plug were observed, which all drinking water was changed every other day $(22,23)$. The control and URSA groups were added with the same content of normal saline. For successful mating, two females and one male were stayed together all night, and females with vaginal plug detected the next morning were recorded as gestational day (GD) 0 . Subsequently, the food intake, activity, vaginal bleeding, and bodyweight of mice in each group were observed daily. The mice were euthanized on GD14. The uterus, embryos, placenta, and decidua were separated, and all the tissues were fixed with $4 \%$ paraformaldehyde and stored at $-80^{\circ} \mathrm{C}$. At the same time, the number of total fetuses and viable fetuses were counted. The fetuses were small and dark, accompanied by necrosis and hemorrhage as absorbed fetuses. The absorption rate of fetuses was calculated as the number of absorbed fetuses/number of total fetuses $\times 100 \%(24,25)$.

\section{H\&E Staining}

The decidual tissues from mice were fixed with $4 \%$ paraformaldehyde for 24-48 hours. Then all samples were dehydrated with gradient alcohol, embedded in paraffin, and cut into $3-\mu \mathrm{m}$ thick sections. Paraffin sections were deparaffinized with xylene and alcohol, stained with hematoxylin for 3 minutes and eosin for 2 minutes, and then mounted with resin. Finally, all sections were observed under the microscope.

\section{Immunohistochemistry Staining}

The paraffin sections of decidua harvested at gestational day (GD) 14 . They were baked in an oven at $60^{\circ} \mathrm{C}$ for 2 hours and then dewaxed with xylene and gradient alcohol. Then the slices were penetrated with $2 \%$ Triton solution for 20 minutes before being repaired with sodium citrate antigen retrieval solution. After blocking with normal goat serum working fluid for $30 \mathrm{~min}$, the slices were incubated with HMGB1(1:400 dilution; Abcam, Cambridge, UK), and NF- $\mathrm{KB}$ (1:500 dilution; Cell Signaling TECHOLOGY, MA, USA) at $4^{\circ} \mathrm{C}$ overnight. Then all samples were incubated with the Streptavidin/Peroxidase HistostainTM -Plus Kits (ZSGB-BIO, Beijing, China), and stained with DAB. The positive cells were observed under a Zeiss (Carl Zeiss AG, Oberkochen, Germany) microscope after the sections were sealed with resin.

\section{Western Blotting Assay}

Mouse decidua and human decidual tissue were extracted with lysis buffer, and the protein concentration was detected with a BCA protein quantification kit (Beyotime, Shanghai, China). The total lysate was separated by $10 \%-12 \%$ sodium dodecyl sulfatepolyacrylamide gel electrophoresis (SDS-PAGE) and transferred to a polyvinylidene fluoride (PVDF) membrane. The membranes were incubated overnight with HMGB1 (1:10000 dilution; Abcam, Cambridge, UK), GSDMD (1:500 dilution; Affinity, USA), caspase-1 (1:1000 dilution; Abcam, Cambridge, UK), NLRP3 (1:200 dilution; NOVUS, USA), TLR2 (1:500 dilution; Abcam, Cambridge, UK), TLR4 (1:500 dilution; Abcam, Cambridge, UK), RAGE (1:1000 dilution; Abcam, Cambridge, $\mathrm{UK})$ antibodies in a shaker at $4^{\circ} \mathrm{C}$ overnight, $\beta$-actin $(1: 1000$ dilution; Abcam, Cambridge, UK) was used as an internal reference control. Membranes were incubated with secondary antibodies for 2 hours after washing the next day, and the ECL detection kit (Pierce Biotechnology, Rockford, USA) was then used for signal detection.

\section{Immunofluorescence Staining}

Subcellular localization of HMGB1 and F4/80 or CD68 proteins in decidua was identified by using immunofluorescent staining. The steps before blocking were the same as immunohistochemistry. After blocking with $10 \%$ donkey serum for 2 hours, they were incubated with HMGB1(1:400 dilution; Abcam, Cambridge, UK), and F4/80 (1:100 dilution; Invitrogen, MA, USA) or CD68(1:100 dilution; GeneTex, CA, USA) at $4^{\circ} \mathrm{C}$ overnight. The next day, sections were allowed to return to room temperature and incubated with anti-rabbit IgG-594 (1:1000 dilution; Jackson, PA, USA) and anti-rat FITC (1:50 dilution; ZSGB-BIO, Beijing, China) for 2 hours. Then stain the cell nucleus with Hoechst 33342 (1:500 dilution; Thermo, USA.) for 5 minutes, and add a fluorescence quencher to observe under a fluorescence microscope (Carl Zeiss AG, Oberkochen, Germany).

\section{ELISA}

Mouse blood was collected by eyeball removal and allowed to stand at room temperature for 2 hours, centrifuged at $3000 \mathrm{rpm}$ for 10 minutes, and then the supernatant was aspirated and stored at $-80^{\circ} \mathrm{C}$ until use. Then the HMGB1 concentration in the mice serum was further detected by the ELISA (CLOUDCLONECORP, Wuhan, China), assay according to the manufacturers' instructions. 


\section{Statistical Analysis}

Statistical analysis was performed using SPSS 22.0 and ImageJ, and the results were expressed as mean \pm SEM. Differences between multiple groups were analyzed using analysis of oneway analysis of variance followed by Bonferroni correction, and the difference between the two groups were compared with the paired $t$-test. Values of $p<0.05$ was considered statistically significant.

\section{RESULTS}

\section{Up-regulation of HMGB1 and Pyroptosis- Associated Proteins in Decidual Tissue of URSA Patients}

Decidual tissue was obtained from patients to investigate the expression of HMGB1 protein and pyroptosis-associated proteins. Consistent with our previous report, the protein expression of HMGB1 in the decidual tissue from the URSA patients (URSA group) was higher than that of the control (normal group) (Figures 1A, B). GSDMD, NLRP-3, and caspase-1 proteins were also up-regulated in the URSA group (Figures 1A, C-E). The above results indicated that HMGB1 and pyroptosis might be associated with URSA.

\section{Inhibition of HMGB1 Attenuated Inflammatory Responses at the Maternal-Fetal Interface in URSA Mice}

To explore the pathogenesis of URSA, we established a mouse model of URSA. The uterus, fetus, and placenta of the three groups of mice were shown in Figure 2A. Compared to control group, some fetuses and placenta in URSA group were found to be small in size and darker in color. Aspirin is utilized as an inhibitor drug against HMGB1. Interestingly, the situation of miscarriage in the aspirin treatment group was better than that in the URSA group. The number of fetuses and embryo resorption rate were shown in Figure $\mathbf{2 B}$ and Table $\mathbf{1}$. The embryo resorption rate was $6.19 \%$ in the control group, $20.00 \%$ in the URSA group, and $7.92 \%$ in the aspirin group. The URSA group had a higher embryo absorption rate than the control group $(p<0.01)$, indicating that the abortion model was successfully constructed. Treatment with aspirin reduced the rate of miscarriage compared to the URSA group $(p<0.05)$. These results indicated that mouse model of URSA was established successful, and aspirin could reduce embryonic absorption rate in URSA group. Meanwhile, H\&E staining showed that the infiltrating cells in the decidual tissue of the URSA group were significantly more than those of the control group, the difference was statistically significant $(p<0.001)$, accompanied by

A

Normal URSA
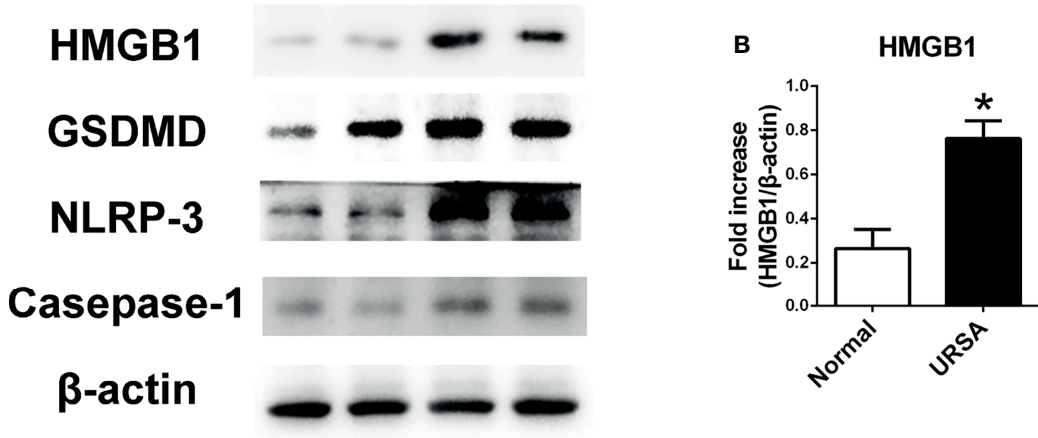

C GSDMD

D

NLRP-3

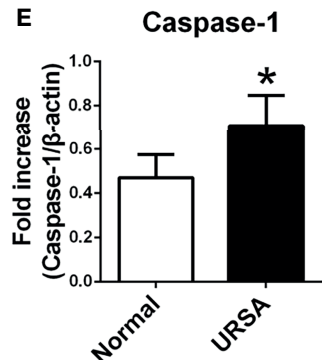

FIGURE 1 | The expression of HMGB1 and pyroptosis-associated proteins GSDMD, NLRP-3, and Caspase-1 in decidual tissue of URSA patients. (A) HMGB1, GSDMD, NLRP-3, and Caspase-1 protein were detected using Western blotting. (B) Quantitative data analysis for HMGB1. (C) Quantitative data analysis for GSDMD. (D) Quantitative data analysis for NLRP-3. (E) Quantitative analysis for Caspase-1. All the results were representatives of three independent experiments and data were expressed as mean \pm SEM. (n=20/group). ${ }^{\star} P<0.05,{ }^{\star \star} P<0.01$ compared to the control. 


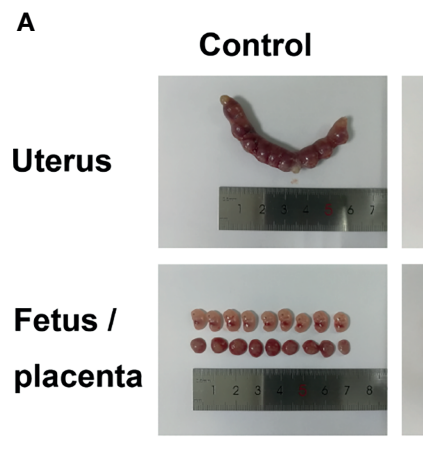

C

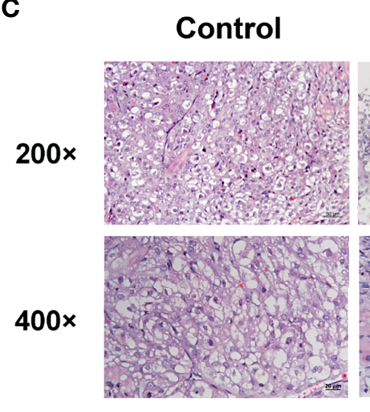

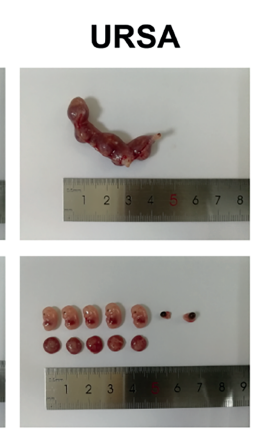

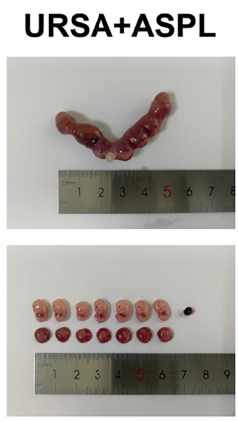

URSA

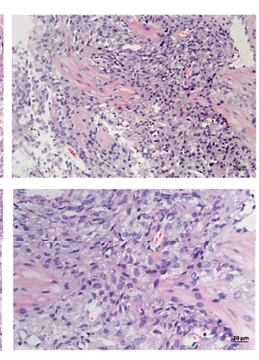

URSA+ASPL

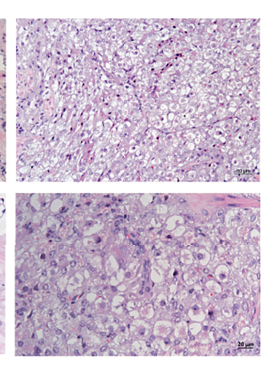

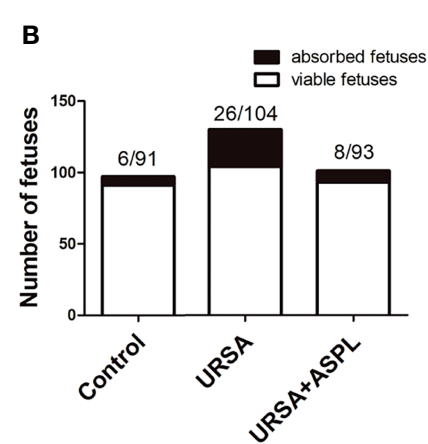

D

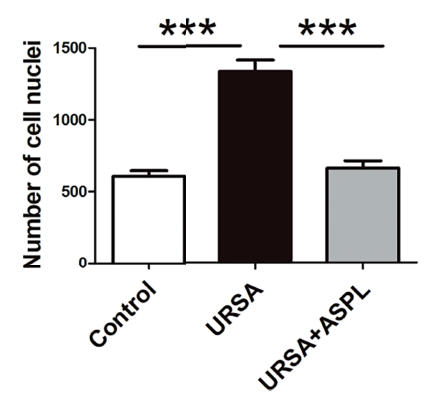

FIGURE 2 | Uterine anatomy and pathological changes of decidua in mice. (A) Left and right uterine horns, fetus, and placenta from normal pregnant mice, aborted mice, and drug-treated mice. (B) The number of fetuses. (C) Decidua of the control group, URSA group, and URSA+ASPL group were stained by H\&E staining. (D) Quantitative assessment of infiltrating cells in decidua by counting the number of cell nuclei in H\&E staining. The results were representatives of three independent experiments and data were expressed as mean \pm SEM. ${ }^{\star \star \star} P<0.001$. Scale bar represents $50 \mu \mathrm{m}$ and $20 \mu \mathrm{m}$. ( $\mathrm{n}=6 /$ group).

disorganized cells and fragmented nuclei (Figures 2C, D). However, aspirin treatment could ameliorate the inflammatory response at the maternal-fetal interface in URSA mice, and the difference was statistically significant $(p<0.001)$.

\section{Treatment With Aspirin Down-Regulated the Level of HMGB1}

Our previous studies have proved that HMGB1 plays an important role in URSA patients (15). To verify the role of HMGB1 in the URSA mouse model, we examined the expression of HMGB1 in the decidual tissues of URSA mice by immunohistochemical staining. As shown in Figures $\mathbf{3 A}, \mathbf{B}$, compared with the control group, not all positive staining in the URSA group was in the nucleus, but there was a large amount of positive staining in the cytoplasm and extracellular. In the aspirin treatment group, HMGB1 was still expressed in the nucleus. The above difference was statistically significant $(p<0.01)$. Biological effects only occur when HMGB1 is expressed outside the nucleus. Western blot detection showed that the expression level of HMGB1 protein was higher in the URSA group than in the control group, and decreased in the aspirin group (Figures $3 \mathrm{C}, \mathrm{D}$ ). These results indicated that HMGB1 was involved in the inflammatory response of URSA decidual tissue, which caused immune disorders. However, aspirin ameliorated HMGB1induced immune disturbance at the maternal-fetal interface in URSA mice. Finally, the levels of HMGB1 in sera of mice in the control group, URSA group, and aspirin group were detected by ELISA (Figure 3E). The results showed that the serum HMGB1 expression level in the URSA group was higher than that in the control group, which was significantly lower after aspirin treatment. It was indicated that aspirin treatment could inhibit the expression of HMGB1 in URSA mice.

TABLE 1 | The absorption rate among the three groups.

\begin{tabular}{lccc}
\hline & Control & URSA & URSA+ASPL \\
\hline Number of animals & 11 & 14 & 12 \\
Number of viable fetuses & 91 & 104 & 93 \\
Number of absorbed fetuses & 6 & 130 & 8 \\
Number of total fetuses & 97 & $26.00 \%(26 / 130)^{\mathrm{a}}$ & 101 \\
Absorption rate of fetuses (\%) & $6.19 \%(6 / 97)$ & $7.92 \%(8 / 101)^{\mathrm{b}}$ \\
\hline
\end{tabular}

Data were analyzed using the Chi-square. ${ }^{a} P<0.01$ compared with the Control group, ${ }^{b} P<0.05$ compared with the URSA group. 
A

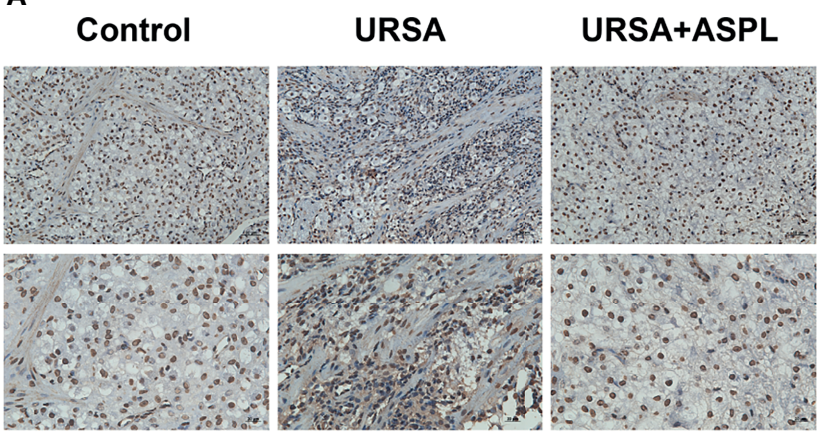

C

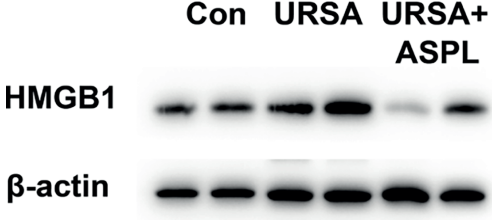

D

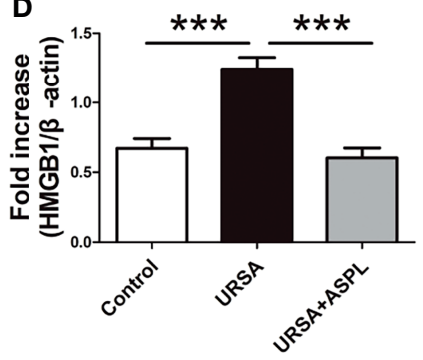

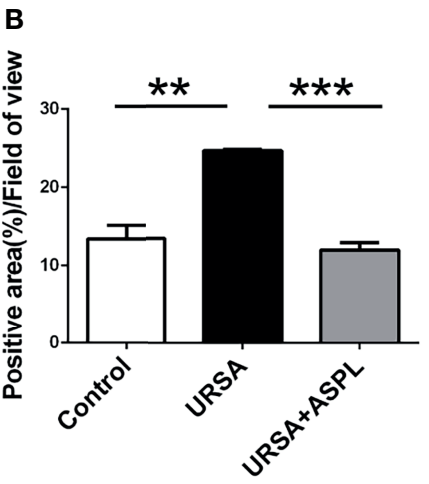

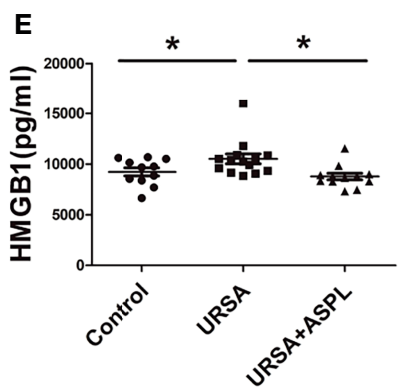

FIGURE 3 | The expression of HMGB1 in mouse decidual tissue. (A) The expression of HMGB1 in mouse decidual tissue was investigated by immunohistochemistry staining. Scale bar represents $50 \mu \mathrm{m}$ and $20 \mu \mathrm{m}$. ( $\mathrm{n}=6 / \mathrm{group}$ ). (B) Quantitative assessment of the HMGB1 positive staining area in decidua using ImageJ software. (C) The protein levels of HMGB1 were identified by Western blot analysis. (D) The quantification data analysis of HMGB1 in the mouse decidual tissues. Data were expressed as mean \pm SEM. ( $n=6$ /group). (E) The serum HMGB1 levels of mice among control group, URSA group, and URSA+ASPL group were analyzed by ELISA. All the results were representatives of three independent experiments and data were expressed as mean $\pm \mathrm{SEM} .{ }^{\star} P<0.05,{ }^{\star \star} P<0.01,{ }^{\star \star \star} P<0.001($ Chart $\mathrm{D} n=11$ in control group; $n=14$ in URSA group; $n=11$ in URSA+ASPL group).

\section{HMGB1 Derived From Decidual Macrophages at the Maternal-Fetal Interface of URSA}

Double immunofluorescence staining was used to verify whether HMGB1 is released by macrophages at the maternal-fetal interface. F4/80 and CD68 are well-known surface-specific markers of macrophages. As shown in Figure $\mathbf{4 A}$, the expression of HMGB1 (red) and F4/80 (green) was higher in the URSA group than that in the control group, and the difference was statistically significant (Figures 4C, D), and there were more HMGB1 and F4/80 double positive cells emerged. It was suggested that macrophage-derived HMGB1 was abundantly expressed in the nucleus as well as in the cytoplasm and extracellular space in the decidual tissue of URSA patients. This was also verified in mouse decidual tissue. As shown in Figure 4B, HMGB1 (red) and CD68 (green) double-positive cells were abundantly expressed in the URSA group, whereas this double-positive cell expression was reduced in the aspirin group, and the difference was statistically significant (Figures 4E, F). These indicated that macrophages in the decidual tissue of URSA could secrete HMGB1 actively, whereas aspirin could down-regulate the expression of macrophage-derived HMGB1.

\section{The Altered HMGB1 Related Receptors and NF-kB Signaling in Abortion Tissue Suffered From URSA}

To investigate whether HMGB1 expressed in decidual tissue was involved in receptors (RAGE/TLRs) and NF- $\kappa B$ intracellular signaling pathways, we measured the protein levels of RAGE, TLR2, and TLR4 in mice of three groups by western blotting. The protein expression of HMGB1 receptors RAGE, TLR2, and TLR4 in the URSA group were significantly up-regulated compared with the control group, while the expression were significantly down-regulated in the aspirin group (Figures 5A-D). Meanwhile, the location of NF- $\mathrm{KB}$ in the three groups was detected by immunohistochemical staining. Low levels of NF$\kappa \mathrm{B}$ p65 were concentrated in the cytoplasm and nucleus in the control group. While in the URSA group, there was an intense immune response to NF- $\mathrm{KB}$ p 65 in both the nucleus and cytoplasm of mice decidua. The aspirin group was similar to the control group (Figure 5E). Therefore, the results showed that the HMGB1 related receptors (RAGE/TLR2/TLR4) were upregulated and $\mathrm{NF}-\kappa \mathrm{B}$ signaling were activated in the decidua tissue of URSA group, and their expression could be downregulated after aspirin intervention. Thus, we suggested that the HMGB1-TLRs/RAGE-NF-KB pathway may be played an 

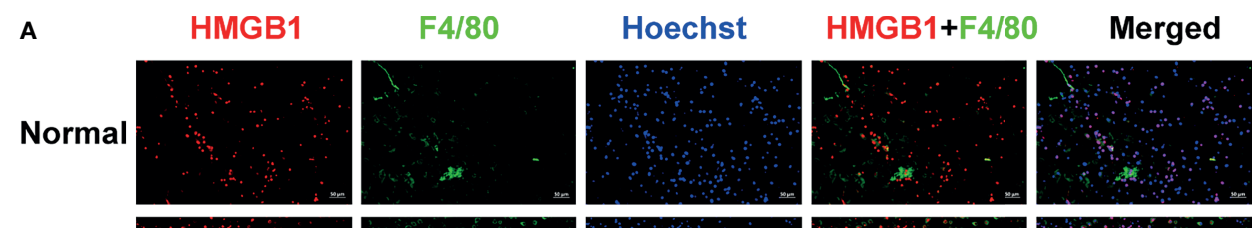

URSA
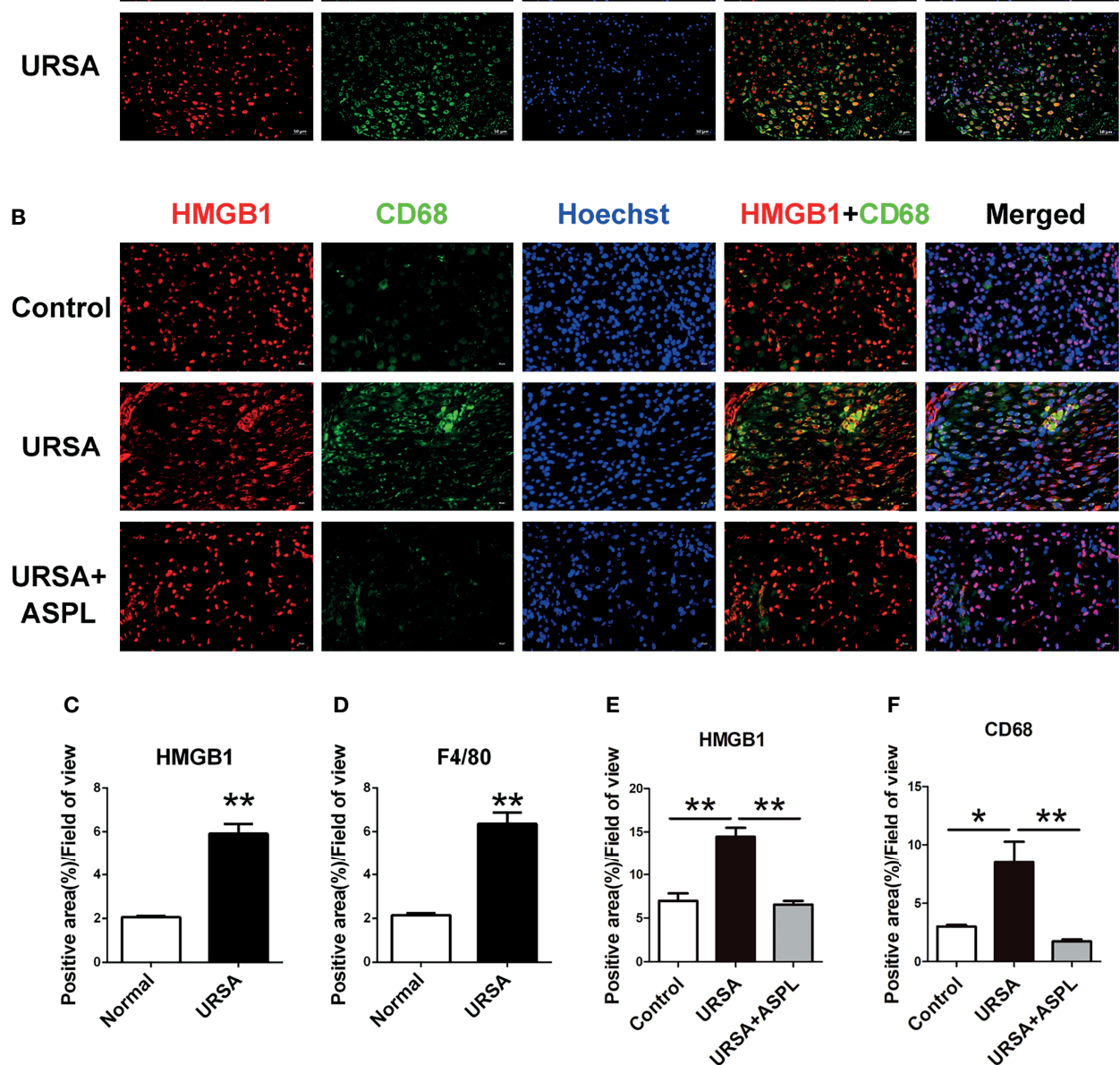

FIGURE 4 | The colocalization between macrophages $\left(\mathrm{F} 4 / 80^{+}\right.$or $\left.\mathrm{CD} 68^{+}\right)$and $\mathrm{HMGB} 1$ at the maternal-fetal interface. Double immunofluorescent staining of F4/80 and HMGB1 in human decidua (A) and CD68 and HMGB1 in mouse decidua (B). Quantitative assessment of the HMGB1 (C) and F4/80 (D) positive staining area in human decidua using ImageJ software. Quantitative assessment of the HMGB1 (E) and CD68 (F) positive staining area in mouse decidua using ImageJ software. HMGB1 was labeled with red fluorescent reagent. F4/80 and CD68 were labeled with green fluorescent reagent. The nucleus was stained blue with Hoechst. Scale bar represents $50 \mu \mathrm{m}$ (human, $n=20 /$ group) and $20 \mu \mathrm{m}$ (mouse, $n=6 /$ group). All the results were representatives of three independent experiments, and data were expressed as mean \pm SEM. ${ }^{\star} P<0.05,{ }^{\star \star} P<0.01$.

important role in the maternal-fetal interface of URSA, and the inhibition of HMGB1 could block this pathway.

\section{Inhibition of HMGB1 Down-Regulated Pyroptosis-Associated Proteins at the Maternal-Fetal Interface in URSA}

To determine the expression of pyroptosis-associated proteins in mouse decidual tissues, we examined the expression of GSDMD, NLRP-3, and caspase-1 with western blot assay. As shown in Figure 6, the expressions of GSDMD, NLRP-3, and caspase-1 were consistent with those in human decidua tissue, and the URSA group had higher expression than that of the control group (Figures 6A-D). However, the expression level of these proteins were down-regulated in the aspirin group. It was suggested that aspirin could intervene in the NLRP3-caspase1GSDMD signaling pathway, and indicating that aspirin could suppress the activation of pyroptosis.

\section{DISCUSSION}

The present study found that HMGB1, GSDMD, NLRP3, and caspase1 proteins were up-regulated in the decidual tissues of humen and mice in the URSA group. Moreover, the infiltrating cells of decidual tissue in the URSA group were significantly more than those in the control group, the cell arrangement was 


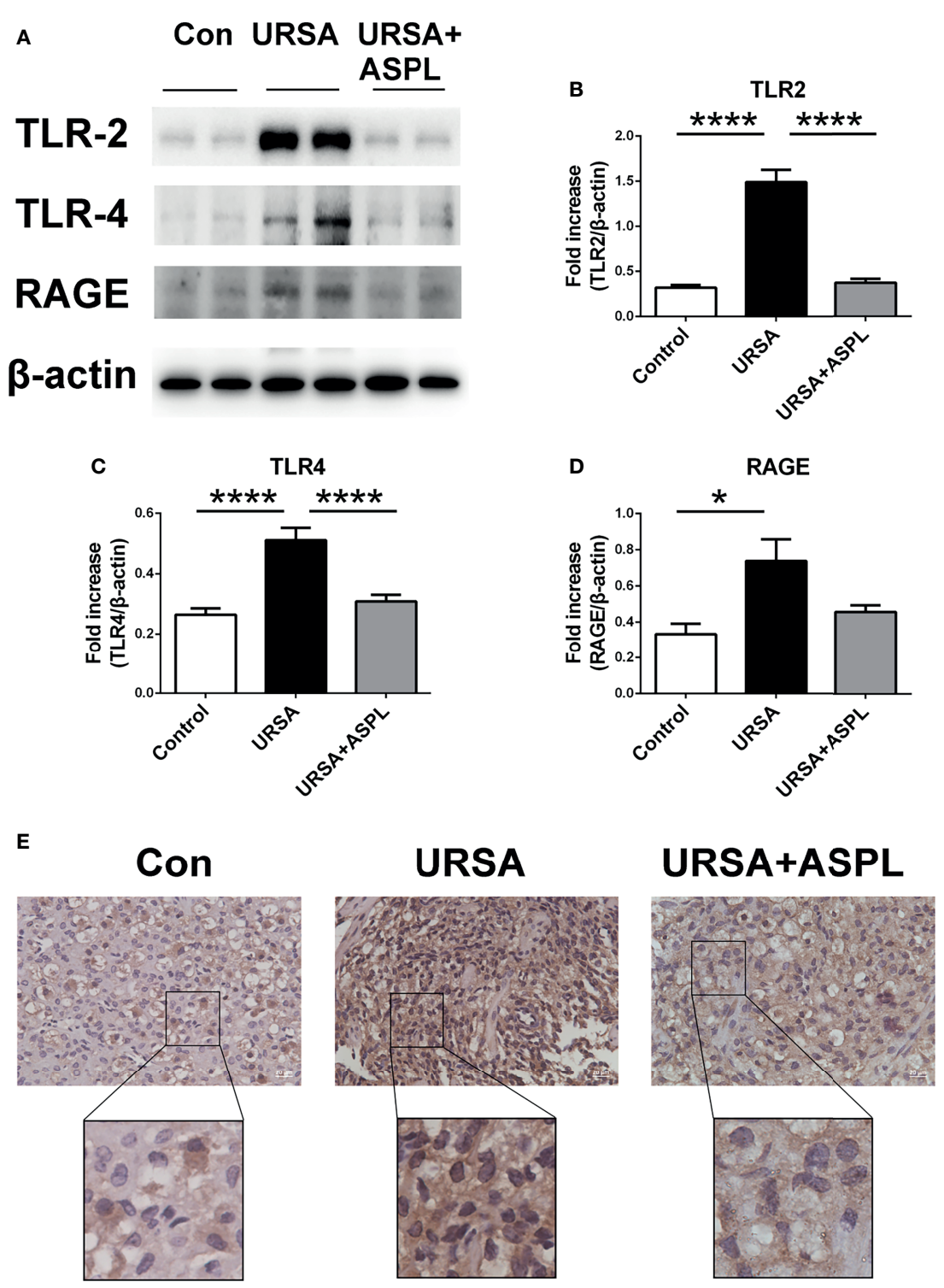

FIGURE 5 | The analysis of TLR2, TLR4, RAGE, and NF-kB p65 in mouse decidua. (A) The protein levels of TLR2, TLR4, and RAGE were identified by Western blot analysis. (B) Quantitative data analysis for TLR2. (C) Quantitative data analysis for TLR4. (D) Quantitative data analysis for RAGE. (n = 6/group). (E) The expression of NF- $\kappa B$ p65 was detected by immunohistochemistry in decidua among control group, URSA group, and URSA+ASPL group. Scale bar represents $20 \mu$ m. ( $n=6 /$ group). All the results were representatives of three independent experiments and data were expressed as mean \pm SEM. ${ }^{\star} P<0.05$, ${ }^{\star \star \star \star} P<0.0001$.

disordered, and the nucleus was broken. We also found that the increased expression of HMGB1 in the URSA group may come from the active secretion of macrophages. The excessive expression of these proteins could be down-regulated after intervention by the HMGB1 inhibitor aspirin. These data clarify the source and possible mechanism of the high expression of HMGB1 in the decidual tissues of the URSA group, suggesting that HMGB1 and pyroptosis-associated proteins play a vital role in the development of URSA.

The studies about URSA have focused on the influence of peripheral blood immune cells. Recent studies found that the imbalance between Treg and Th17 cells may be related to the pathogenesis of URSA (26), and found that the increase in Th17related cytokine levels may be one of the related factors of URSA (27). Moreover, the data suggested that the imbalance of the Treg/Th17 ratio may be regulated through HMGB1, and HMGB1 was positively correlated with Th cell-related factors, and negatively correlated with Treg cell-related cytokines (28). HMGB1 structurally contains three functional regions: A box, B box, and acidic C-terminus, and the $\mathrm{B}$ box is a functional structural area with two key binding sites for RAGE receptor and Toll-like receptors that play an important role in the inflammatory response (10). The relationship between HMGB1 and diseases has attracted widespread attention, including acute 

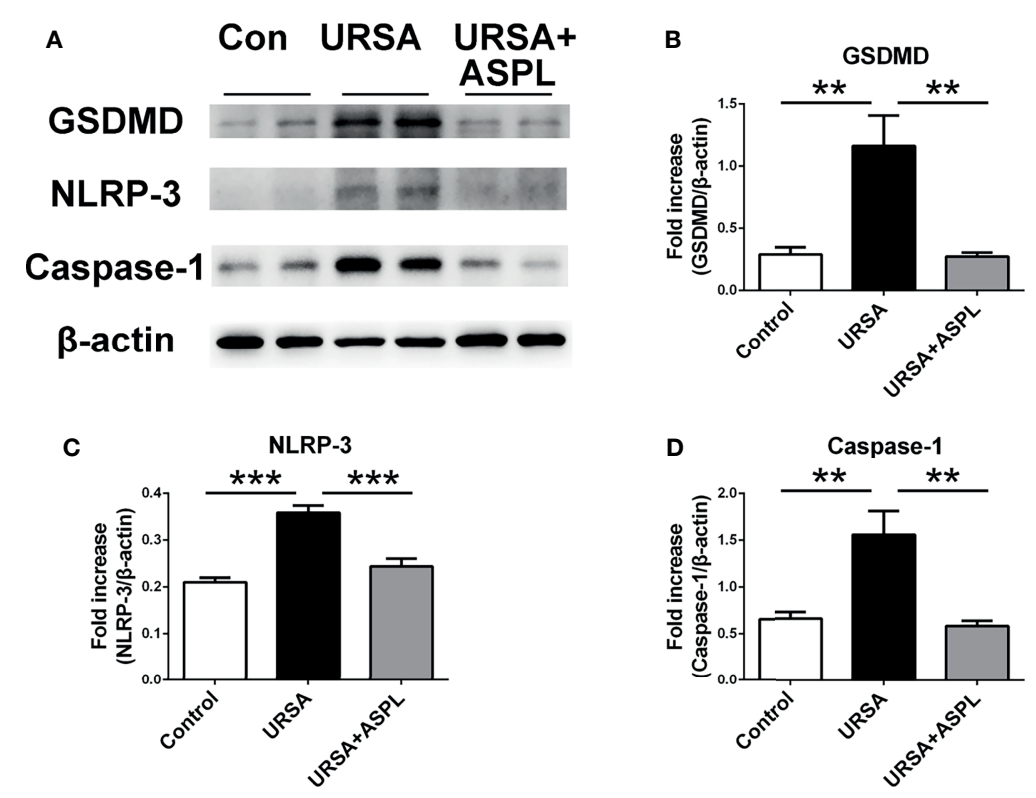

FIGURE 6 | The analysis of GSDMD, NLRP-3, and Caspase-1 in mouse decidua. (A) The protein levels of GSDMD, NLRP-3, and Caspase-1 were identified by Western blot analysis. (B) Quantitative data analysis for GSDMD. (C) Quantitative data analysis for NLRP-3. (D) Quantitative data analysis for Caspase-1. All the results were representatives of three independent experiments and data were expressed as mean $\pm \mathrm{SEM}$. ${ }^{\star \star} P<0.01$, ${ }^{\star \star \star} P<0.01$. (n $=6 /$ group).

injury-related inflammatory responses, tumors, organ transplants, and autoimmune diseases (29-32). Studies have found that the expression of HMGB1 protein was increased in the villi of RPL patients (14). Our experiment also found that the expression level of HMGB1 was up-regulated in the decidua tissue of URSA patients. These data revealed that HMGB1 may have an association with the pathogenesis of URSA. In addition, we also found that GSDMD, NLRP3, and caspase1 proteins were up-regulated in the decidual tissues of URSA patients, indicating the pathogenesis of URSA may also be related to pyroptosisassociated proteins.

HMGB1 is essential for early pregnancy events before and during blastocyst implantation (12). In the early pregnancy, HMGB1 has been shown to play a key role in driving the proinflammatory response during embryo implantation (16). Exogenous and endogenous HMGB1 initiate an inflammatory response at the time of embryo implantation, high HMGB1 expression around the implantation site, and stromal cell proliferation and differentiation required to induce uterine cell decidualization (13). Several studies have reported that HMGB1 levels are elevated in patients with pregnancy complicated by gestational diabetes mellitus, preeclampsia, and preterm prelabor rupture of membranes (33-35). Our previous results indicated that HMGB1 was highly expressed at the maternal-fetal interface of URSA patients $(15,20)$. Our study found disorganized cells and cellular infiltration in the mouse decidual tissue of the URSA group, indicating the mouse decidua tissue was in a state of inflammation. However, as a DAMP molecule, HMGB1 plays an important role in proinflammatory (36). Our research results showed that HMGB1 was abundant in the cytoplasm and extracellular in the decidua tissue of the URSA group. Thus, the inflammation at the mouse maternal-fetal interface was induced by HMGB1.

This research indicated that the high level of HMGB1 during pregnancy may induce excessive or continuous inflammation, which will lead to unfavorable pregnancy outcomes at this critical stage of pregnancy. Moreover, during pregnancy, the induction and maintenance of immune tolerance is a prerequisite for a successful pregnancy, involving the participation of a variety of immune cells, such as T cells, NK cells, macrophages, and so on (37). Previous studies have found that decidual immune cells at the URSA maternal-fetal interface play a crucial role in regulating immune tolerance (20). Macrophages can secrete and increase the expression level of HMGB1 in inflammatory response (38). To further investigated the relationship between macrophages and HMGB1, we performed double immunofluorescence staining experiments and found that HMGB1 colocalized with F4/80 or CD68, a surface-specific marker of macrophages, in both human and mouse decidual tissues. Meanwhile, HMGB1 is mainly found in the nucleus and can be actively or passively secreted into the extracellular space to play a pro-inflammatory role (10). Our study indicated that the highly expressed HMGB1 in the URSA group was actively secreted by macrophages.

Previous research found that HMGB1 can be released from immune cells to the extracellular space, increasing sterile inflammation and expanding tissue damage (15), and proposed that it may be regulated by the HMGB1-RAGE/TLR2/TLR4-NF$\kappa \mathrm{B}$ signaling pathway (15). Studies have showed that HMGB1 can be caused acute lung injury by activating inflammasomes in macrophages and was related to TLR2, TLR4, and RAGE/NF- $\kappa B$ signaling pathways (38) HMGB1 can be used as a potential target 
for bone marrow mesenchymal stem cells to treat multiple organ dysfunction syndromes. The possible mechanism was that HMGB1 may regulate the TLR2, TLR4-mediated NF- $\kappa B$ signal pathway (39). It is well known that HMGB1 can interact with RAGE and Toll-like receptors to participate in cell signal transduction (11). Studies had shown that the TLR4/NF-KB signaling pathway plays an important role in placental inflammation (40). Similarly, the up-regulation of HMGB1 and its receptors RAGE, TLR2, and TLR4 in the URSA group were found in this study, which may suggest that high expression of HMGB1 can induce an increase in the expression of RAGE and Toll-like receptors. Moreover, in the URSA group, there was a strong positive reaction to NF- $\kappa \mathrm{B}$ protein in the nucleus and cytoplasm of the decidual tissue, indicating HMGB1 may stimulate the activation of NF- $\kappa \mathrm{B}$ through its receptor on URSA.

The activation of the NLRP-3 inflammasome and the increased expression of caspase- 1 protein were found in the decidua and chorionic tissues of missed abortion (41). High expression of the NLRP-3 inflammasome was also found at the maternal-fetal interface of patients with recurrent spontaneous abortion, promoting pro-caspase- 1 accumulation and division into the activated form caspase-1 when NLRP-3 recognizes stimulatory signals (damage-associated molecular patterns [DAMPs]) in vitro or in vivo, and then activate IL-1 $\beta$, IL-18 to participate in subsequent inflammation (42). However, NLRP-3 and caspase-1 are the main participants in the process of pyroptosis, which is a type of programmed cell death closely related to inflammation, mediated by Gasdermin protein, and dependent on caspase activity (43). Moreover, there was research shown that HMGB1 could induce hepatocyte pyroptosis leading to aseptic inflammation and liver damage (19). In this study, HMGB1, NLRP-3, caspase-1, and GSDMD expression levels were up-regulated in the decidua of URSA patients. Meanwhile, these molecules were also highly expressed in the mouse decidua. It was speculated that HMGB1 could induce pyroptosis leading to the onset of URSA.

In recent years, HMGB1 has been widely used as a therapeutic target for diseases (44). HMGB1 was a key pro-inflammatory factor in acute lung injury, involved in the pathogenesis of acute lung injury by inducing M1 polarization through TLR2- and TLR4-mediated NF- $\kappa \mathrm{B}$ signaling pathways in macrophages (45). In addition, in hepatic ischemia-reperfusion injury, octreotide and melatonin could reduce the inflammasome-induced pyroptosis by inhibiting the TLR4-NF-KB-NLRP3 pathway (46). Our study found that HMGB1 and their receptors (RAGE, TLR2, TLR4), pyroptosis-associated proteins (NLRP-3, caspase-1, GSDMD), and NF-kB were highly expressed in tissues of unexplained recurrent miscarriage. Besides, studies have shown that taking aspirin for a short period can reduce the level of extracellular HMGB1 (47). Salicylic acid, the hydrolysis product of aspirin in vivo, can be used as an effective inhibitor of HMGB1 and applied in the treatment of various diseases such as tumors $(48,49)$. This hinted that aspirin can be used as a specific inhibitor of HMGB1. Besides, aspirin is a commonly used drug in assisted reproductive technology, especially the treatment of recurrent miscarriage. In our study, mice treated with aspirin reduced their embryonic absorption rate, suggesting the treatment effect of aspirin on URSA. Moreover, our

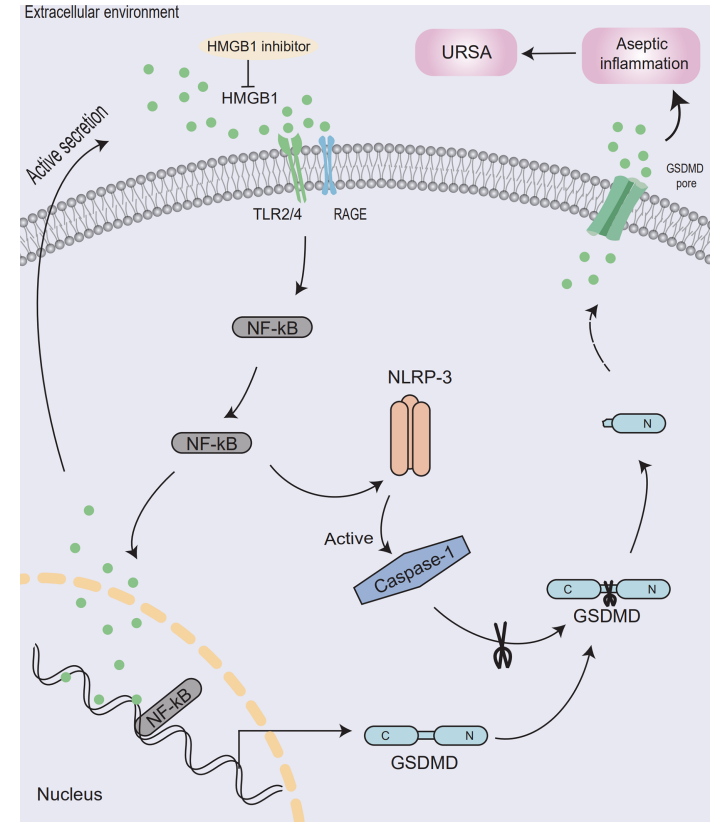

FIGURE 7 | Model of aseptic inflammation caused by HMGB1-mediated pyrolysis in decidua tissue of unexplained recurrent spontaneous abortion. At the maternal-fetal interface, macrophages actively secrete HMGB1 and activate the NF- $\mathrm{kB}$ signaling pathway through its receptor. Then NLRP-3 inflammasome will be assembled, activate the caspase- 1 protein, and release inflammatory factors such as HMGB1, causing aseptic inflammation at the maternal-fetal interface and leading to the development of URSA. Meanwhile, HMGB1 inhibitor could ameliorates the maternal-fetal interface destruction in unexplained recurrent spontaneous abortion.

previous study found that low-dose aspirin can reduce the level of HMGB1 in the plasma of URSA patients (15). It was further verified in mouse experiments that the expression level of HMGB1 in the decidua tissue and peripheral blood of the aspirin treatment group was down-regulated. In addition, the expression of RAGE, TLR2, and TLR4 receptors was lower in URSA+ASPL group than that of the URSA group. This study proved that HMGB1 as a therapeutic target of aspirin could down-regulate the level of HMGB1 and its receptors. Meanwhile, this study shown that in the aspirin intervention group, the levels of pyroptosis-associated molecules were down-regulated compared with the URSA group. Therefore, based on previous studies we suggested that HMGB1 might induce pyroptosis through its receptor and NF- $\kappa \mathrm{B}$ signaling pathway, cause sterile inflammation, and expand the decidual inflammatory response to mediate the development of URSA, while this inflammatory response can be intervened by the HMGB1 blocker aspirin.

In conclusion, the possible mechanism was that HMGB1 entered cells through its receptor and activates $N F-\kappa B$ signaling pathway. Then, pyroptosis would be activated, which induced aseptic inflammation, ultimately leading to the destruction of the maternal-fetal interface and the development of URSA (Figure 7). Moreover, aspirin had a therapeutic effect 
on unexplained recurrent miscarriage. This was the first time to explore the application of HMGB1 as a therapeutic target of aspirin in URSA disease, providing a theoretical basis for the treatment of clinical URSA patients.

\section{DATA AVAILABILITY STATEMENT}

The original contributions presented in the study are included in the article/supplementary material. Further inquiries can be directed to the corresponding authors.

\section{ETHICS STATEMENT}

The studies involving human participants were reviewed and approved by the Medical Ethics Committee of the First Affiliated Hospital of Anhui Medical University. The patients/participants provided their written informed consent to participate in this study. The animal study was reviewed and approved by the Experimental Animal Ethics Committee of Anhui Medical University. The animal study was reviewed and approved by the Experimental Animal Ethics Committee of Anhui Medical University (ethics number: LLSC20201138).

\section{AUTHOR CONTRIBUTIONS}

$\mathrm{XX}, \mathrm{YC}, \mathrm{XW}$, and HZ designed the study. DZ and HZ performed the experiments and analyzed the data. DZ, HZ, JL, JW, and CM bled the mice and samples acquired. JY and XP helped for language checking. JW, CM, DL, YY, YR, ZZ, and PZ helped for human samples acquired. DZ and HZ wrote the paper. All authors read and approved the final manuscript.

\section{REFERENCES}

1. Bender Atik R, Christiansen OB, Elson J, Kolte AM, Lewis S, Middeldorp S, et al. ESHRE Guideline: Recurrent Pregnancy Loss. Hum Reprod Open (2018) 2:hoy004. doi: 10.1093/hropen/hoy004

2. Practice Committee of the American Society for Reproductive Medicine. Definitions of Infertility and Recurrent Pregnancy Loss: A Committee Opinion. Fertil Steril (2020) 3:533-35. doi: 10.1016/j.fertnstert.2019.11.025

3. de Ziegler D, Frydman RF. Recurrent Pregnancy Losses, a Lasting Cause of Infertility. Fertil Steril (2021) 3:531-32. doi: 10.1016/j.fertnstert.2020.12.004

4. Hennessy M, Dennehy R, Meaney S, Linehan L, Devane D, Rice R, et al. Clinical Practice Guidelines for Recurrent Miscarriage in High-Income Countries: A Systematic Review. Reprod BioMed Online (2021) 42(6):114671. doi: 10.1016/j.rbmo.2021.02.014

5. Rai R, Regan L. Recurrent Miscarriage. Lancet (2006) 368(9535):601-11. doi: 10.1016/s0140-6736(06)69204-0

6. Gao Y, Wang PL. Increased CD56(+) NK Cells and Enhanced Th1 Responses in Human Unexplained Recurrent Spontaneous Abortion. Genet Mol Res (2015) 14(4):18103-9. doi: 10.4238/2015.December.22.36

7. Tafuri A, Alferink J, Möller P, Hämmerling GJ, Arnold B. T Cell Awareness of Paternal Alloantigens During Pregnancy. Science (1995) 270(5236):630-3. doi: $10.1126 /$ science.270.5236.630

8. Cramer DW, Wise LA. The Epidemiology of Recurrent Pregnancy Loss. Semin Reprod Med (2000) 18(4):331-9. doi: 10.1055/s-2000-13722

\section{FUNDING}

The work was supported by grants from the National Natural Sciences Foundation of China (No. 32000642 and 82000399), the Natural Science Foundation of Anhui Province (No.1908085MH244), the Natural Science Foundation of the Anhui Higher Education Institution (No. KJ2019A0285), the Nonprofit Central Research Institute Fund of Chinese Academy of Medical Sciences (No. 2019PT310002), the Research Fund of Anhui Institute of translational medicine (ZHYX2020A001).

\section{ACKNOWLEDGMENTS}

This study was supported by Special Funds for Development of Science and Technology of Anhui Province, YDZX20183400004194, Research Fund Project granted from Anhui Research Institute of Translational Medicine, 2017zhyx30, and National Major Scientific Research Instrument and Equipment Development Project, 11627803. The authors wish to acknowledge all the staff of the IVF center at the First Affiliated Hospital of Anhui Medical University for their support for this study. We thank DDS, PY and LW in the Center of Cryo-Electron Microscopy (CCEM), Zhejiang University for their technical assistance on Scanning Electron Microscopy, and the Beijing Genomics Institute (BGI, Shenzhen, China) for the technical assistance on sc-RNA sequencing. We express thanks to Junqiang Zhang, Zhen $\mathrm{Yu}$, and Huiru Chen from Anhui Province Key Laboratory of Reproductive Health and Genetics, Anhui Medical University, for experimental instruction. We also express thanks to Hua Wang from the Department of Toxicology, School of Public Health, Anhui Medical University, for experimental instruction. We thank Tang Dongdong from the Reproductive Center of the First Affiliated Hospital of Anhui Medical University for his antibody support.

9. Martinotti S, Patrone M, Ranzato E. Emerging Roles for HMGB1 Protein in Immunity, Inflammation, and Cancer. Immunotargets Ther (2015) 4:101-9. doi: $10.2147 /$ itt.S58064

10. Yuan SL, Liu ZP, Xu ZR, Liu J, Zhang J. High Mobility Group Box 1 (HMGB1): A Pivotal Regulator of Hematopoietic Malignancies. J Hematol Oncol (2020) 13(1):91. doi: 10.1186/s13045-020-00920-3

11. Kaur I, Behl T, Bungau S, Kumar A, Mehta V, Setia D, et al. Exploring the Therapeutic Promise of Targeting HMGB1 in Rheumatoid Arthritis. Life Sci (2020) 258:118164. doi: 10.1016/j.lfs.2020.118164

12. Aikawa S, Deng WB, Liang XH, Yuan J, Bartos A, Sun XF, et al. Uterine Deficiency of High-Mobility Group Box-1 (HMGB1) Protein Causes Implantation Defects and Adverse Pregnancy Outcomes. Cell Death Differ (2020) 27(5):1489-504. doi: 10.1038/s41418-019-0429-Z

13. Saito Reis CA, Padron JG. Norman Ing ND and Kendal-Wright CE. HighMobility Group Box 1 Is a Driver of Inflammation Throughout Pregnancy. Am J Reprod Immunol (2021) 85(1):e13328. doi: 10.1111/aji.13328

14. Jin H, Wu J, Yang QH, Cai Y, He WX, Liu CY. High Mobility Group Box 1 Protein Polymorphism Affects Susceptibility to Recurrent Pregnancy Loss by Up-Regulating Gene Expression in Chorionic Villi. J Assist Reprod Genet (2015) 32(7):1123-8. doi: 10.1007/s10815-015-0493-3

15. Zou HJ, Yin JQ, Zhang ZG, Xiang HF, Wang J, Zhu DM, et al. Destruction in Maternal-Fetal Interface of URSA Patients via the Increase of the HMGB1RAGE/TLR2/TLR4-NF- $\kappa B$ Signaling Pathway. Life Sci (2020) 250:117543. doi: $10.1016 /$ j.lfs.2020.117543 
16. Bhutada S, Basak T, Savardekar L, Katkam RR, Jadhav G, Metkari SM, et al. High Mobility Group Box 1 (HMGB1) Protein in Human Uterine Fluid and Its Relevance in Implantation. Hum Reprod (2014) 29(4):763-80. doi: 10.1093/humrep/det461

17. Loveless R, Bloomquist R, Teng Y. Pyroptosis at the Forefront of Anticancer Immunity. J Exp Clin Cancer Res (2021) 40(1):264. doi: 10.1186/s13046-021-02065-8

18. Al Mamun A, Mimi AA, Aziz MA, Zaeem M, Ahmed T, Munir F, et al. Role of Pyroptosis in Cancer and Its Therapeutic Regulation. Eur J Pharmacol (2021) 910:174444. doi: 10.1016/j.ejphar.2021.174444

19. Geng Y, Ma Q, Liu YN, Peng N, Yuan FF, Li XG, et al. Heatstroke Induces Liver Injury via IL-1 $\beta$ and HMGB1-Induced Pyroptosis. J Hepatol (2015) 63 (3):622-33. doi: 10.1016/j.jhep.2015.04.010

20. Wang J, Zhu DM, Yin JQ, Ma C, Peng XQ, Zou HJ, et al. Upregulated HMGB1 Levels in Maternal-Fetal Interface of Patients With Unexplained Recurrent Spontaneous Abortion From Different Sources. J Matern Fetal Neonatal Med (2021), 1-8. doi: 10.1080/14767058.2021.1918084

21. Venereau E, De Leo F, Mezzapelle R, Careccia G, Musco G, Bianchi ME. HMGB1 as Biomarker and Drug Target. Pharmacol Res (2016) 111:534-44. doi: $10.1016 /$ j.phrs.2016.06.031

22. Bulckaen H, Prévost G, Boulanger E, Robitaille G, Roquet V, Gaxatte C, et al. Low-Dose Aspirin Prevents Age-Related Endothelial Dysfunction in a Mouse Model of Physiological Aging. Am J Physiol Heart Circ Physiol (2008) 294(4): H1562-70. doi: 10.1152/ajpheart.00241.2007

23. Doridot L, Passet B, Méhats C, Rigourd V, Barbaux S, Ducat A, et al. Preeclampsia-Like Symptoms Induced in Mice by Fetoplacental Expression of STOX1 are Reversed by Aspirin Treatment. Hypertension (2013) 61 (3):662-8. doi: 10.1161/hypertensionaha.111.202994

24. Hosseini MS, Ali-Hassanzadeh M, Nadimi E, Karbalay-Doust S, Noorafshan A, Gharesi-Fard B. Stereological Study of the Placental Structure in AbortionProne Mice Model (CBA/J×DBA/2J). Ann Anat (2020) 230:151508. doi: 10.1016/j.aanat.2020.151508

25. Yi XC, Zhang J, Liu HX, Yi TX, Ou YH, Liu ML, et al. Suppressed Immune-Related Profile Rescues Abortion-Prone Fetuses: A Novel Insight Into the CBA/J × DBA/2J Mouse Model. Reprod Sci (2019) 26(11):1485-92. doi: 10.1177/1933719119828042

26. Zhu LQ, Chen H, Liu ML, Yuan Y, Wang ZH, Chen Y, et al. Treg/Th17 Cell Imbalance and IL-6 Profile in Patients With Unexplained Recurrent Spontaneous Abortion. Reprod Sci (2017) 24(6):882-90. doi: 10.1177/1933719116670517

27. Roomandeh N, Saremi A, Arasteh J, Pak F, Mirmohammadkhani M, Kokhaei P, et al. Comparing Serum Levels of Th17 and Treg Cytokines in Women With Unexplained Recurrent Spontaneous Abortion and Fertile Women. Iran J Immunol (2018) 15(1):59-67.

28. Ding JW, Zheng XX, Zhou T, Tong XH, Luo CY, Wang XA. HMGB1Modulates the Treg/Th17 Ratio in Atherosclerotic Patients. J Atheroscler Thromb (2016) 23(6):737-45. doi: 10.5551/jat.31088

29. Rao JH, Cheng F, Zhou HM, Yang WJ, Qiu JN, Yang C, et al. Nogo-B Is a Key Mediator of Hepatic Ischemia and Reperfusion Injury. Redox Biol (2020) 37:101745. doi: 10.1016/j.redox.2020.101745

30. Khambu B, Hong HH, Liu S, Liu G, Chen XY, Dong Z, et al. The HMGB1RAGE Axis Modulates the Growth of Autophagy-Deficient Hepatic Tumors. Cell Death Dis (2020) 11(5):333. doi: 10.1038/s41419-020-2536-7

31. Ming BX, Gao M, Zou HJ, Chen HY, Sun Y, Xiao YF, et al. HMGB1 Blockade Differentially Impacts Pulmonary Inflammation and Defense Responses in Poly(I:C)/LPS-Exposed Heart Transplant Mice. Mol Immunol (2016) 76:80-9. doi: 10.1016/j.molimm.2016.06.011

32. Chu YJ, Jing YL, Zhao XY, Wang MR, Zhang ML, Ma R, et al. Modulation of the HMGB1/TLR4/NF- $\mathrm{KB}$ Signaling Pathway in the CNS by Matrine in Experimental Autoimmune Encephalomyelitis. J Neuroimmunol (2021) 352:577480. doi: 10.1016/j.jneuroim.2021.577480

33. Giacobbe A, Granese R, Grasso R, Salpietro V, Corrado F, Giorgianni G, et al. Association Between Maternal Serum High Mobility Group Box 1 Levels and Pregnancy Complicated by Gestational Diabetes Mellitus. Nutr Metab Cardiovasc Dis (2016) 26(5):414-8. doi: 10.1016/j.numecd.2016.02.007

34. Zhong M, Peng JL, Xiang LH, Yang XH, Wang XH, Zhu YB. Epigallocatechin Gallate (EGCG) Improves Anti-Angiogenic State, Cell Viability, and HypoxiaInduced Endothelial Dysfunction by Downregulating High Mobility Group Box 1 (HMGB1) in Preeclampsia. Med Sci Monit (2020) 26:e926924. doi: $10.12659 / \mathrm{msm} .926924$

35. Bredeson S, Papaconstantinou J, Deford JH, Kechichian T, Syed TA, Saade GR, et al. HMGB1 Promotes a P38mapk Associated Non-Infectious
Inflammatory Response Pathway in Human Fetal Membranes. PloS One (2014) 9(12):e113799. doi: 10.1371/journal.pone.0113799

36. Wang SM, Zhang Y. HMGB1 in Inflammation and Cancer. J Hematol Oncol (2020) 13(1):116. doi: 10.1186/s13045-020-00950-x

37. Oreshkova T, Dimitrov R, Mourdjeva M. A Cross-Talk of Decidual Stromal Cells, Trophoblast, and Immune Cells: A Prerequisite for the Success of Pregnancy. Am J Reprod Immunol (2012) 68(5):366-73. doi: 10.1111/j.1600-0897.2012.01165.x

38. Wang J, Li RT, Peng ZY, Hu B, Rao X, Li JG. HMGB1 Participates in LPS -induced Acute Lung Injury by Activating the AIM2 Inflammasome in Macrophages and Inducing Polarization of M1 Macrophages via TLR2, TLR4, and RAGE/Nf-kb Signaling Pathways. J Mol Med (2020) 45(1):61-80. doi: 10.3892/ijmm.2019.4402

39. Xiu GH, Sun J, Li XL, Jin H, Zhu YC, Zhou X, et al. The Role of HMGB1 in BMSC Transplantation for Treating MODS in Rats. Cell Tissue Res (2018) 373 (2):395-406. doi: 10.1007/s00441-018-2823-0

40. Olmos-Ortiz A, Déciga-García M, Preciado-Martínez E, Bermejo-Martínez L, Flores-Espinosa P, Mancilla-Herrera I, et al. Prolactin Decreases LPS-Induced Inflammatory Cytokines by Inhibiting TLR-4/Nfkb Signaling in the Human Placenta. Mol Hum Reprod (2019) 25(10):660-67. doi: 10.1093/molehr/gaz038

41. Zhang X, Zhang K, Zhang Y. Pigment Epithelium-Derived Factor Facilitates NLRP3 Inflammasome Activation Through Downregulating Cytidine Monophosphate Kinase 2: A Potential Treatment Strategy for Missed Abortion. Int J Mol Med (2020) 45(5):1436-46. doi: 10.3892/ijmm.2020.4517

42. Gao P, Zha Y, Gong X, Qiao FY, Liu HY. The Role of Maternal-Foetal Interface Inflammation Mediated by NLRP3 Inflammasome in the Pathogenesis of Recurrent Spontaneous Abortion. Placenta (2020) 101:22129. doi: $10.1016 /$ j.placenta.2020.09.067

43. Jia C, Zhang J, Chen HW, Zhuge YZ, Chen HQ, Qian FY, et al. Endothelial Cell Pyroptosis Plays an Important Role in Kawasaki Disease via HMGB1/ RAGE/cathespin B Signaling Pathway and NLRP3 Inflammasome Activation. Cell Death Dis (2019) 10(10):778. doi: 10.1038/s41419-019-2021-3

44. Xue JM, Suarez JS, Minaai M, Li SJ, Gaudino G, Pass HI, et al. HMGB1 as a Therapeutic Target in Disease. J Cell Physiol (2021) 236(5):3406-19. doi: $10.1002 /$ jcp.30125

45. Li R, Shang Y, Yu Y, Zhou T, Xiong W, Zou XJ. High-Mobility Group Box 1 Protein Participates in Acute Lung Injury by Activating Protein Kinase R and Inducing M1 Polarization. Life Sci (2020) 246:117415. doi: 10.1016/j.lfs.2020.117415

46. El-Sisi AE, Sokar SS, Shebl AM, Mohamed DZ, Abu-Risha SE. Octreotide and Melatonin Alleviate Inflammasome-Induced Pyroptosis Through Inhibition of TLR4-NF-kB-NLRP3 Pathway in Hepatic Ischemia/Reperfusion Injury. Toxicol Appl Pharmacol (2021) 410:115340. doi: 10.1016/j.taap.2020.115340

47. Goetzl EJ, Goetzl L, Karliner JS, Tang N, Pulliam L. Human Plasma PlateletDerived Exosomes: Effects of Aspirin. FASEB J (2016) 30(5):2058-63. doi: 10.1096/fj.201500150R

48. Yang H, Pellegrini L, Napolitano A, Giorgi C, Jube S, Preti A, et al. Aspirin Delays Mesothelioma Growth by Inhibiting HMGB1-Mediated Tumor Progression. Cell Death Dis (2015) 6(6):e1786. doi: 10.1038/cddis.2015.153

49. Choi HW, Tian MY, Song F, Venereau E, Preti A, Park SW, et al. Aspirin's Active Metabolite Salicylic Acid Targets High Mobility Group Box 1 to Modulate Inflammatory Responses. Mol Med (2015) 21(1):526-35. doi: 10.2119/molmed.2015.00148

Conflict of Interest: The authors declare that the research was conducted in the absence of any commercial or financial relationships that could be construed as a potential conflict of interest.

Publisher's Note: All claims expressed in this article are solely those of the authors and do not necessarily represent those of their affiliated organizations, or those of the publisher, the editors and the reviewers. Any product that may be evaluated in this article, or claim that may be made by its manufacturer, is not guaranteed or endorsed by the publisher.

Copyright (C) 2021 Zhu, Zou, Liu, Wang, Ma, Yin, Peng, Li, Yang, Ren, Zhang, Zhou, Wang, Cao and Xu. This is an open-access article distributed under the terms of the Creative Commons Attribution License (CC BY). The use, distribution or reproduction in other forums is permitted, provided the original author(s) and the copyright owner(s) are credited and that the original publication in this journal is cited, in accordance with accepted academic practice. No use, distribution or reproduction is permitted which does not comply with these terms. 